\title{
Posterior resection via atlantoaxial lamina space without internal fixation for C1-C2 intraspinal tumors: A novel approach
}

\section{Beiping Ouyang}

Southern Medical University; General Hospital of Southern Theatre Command of PLA

\section{Xiaobao Zou}

Southern Medical University;General Hospital of Southern Theatre Command of PLA

\section{Ling Ni}

Guangzhou University of Chinese Medicine;General Hospital of Southern Theatre Command of PLA

\section{Su Ge}

Guangzhou University of Traditional Chinese Medicine;General Hospital of Southern Theatre Command ofPLA

\section{Yuyue Chen}

General Hospital of Southern Theatre Command of PLA

\section{Shuang Zhang}

General Hospital of southern Theare Command of PLA

\section{Hong Xia}

General Hospital of Southern Theatre Command of PLA

Xiangyang Ma ( $\sim$ maxy1001@126.com )

Department of Orthopedics, General Hospital of Southern Theatre Command of PLA

\section{Research article}

Keywords: Posterior resection, Atlantoaxial lamina space, Intraspinal tumor

Posted Date: October 15th, 2020

DOl: https://doi.org/10.21203/rs.3.rs-64404/v2

License: (c) (1) This work is licensed under a Creative Commons Attribution 4.0 International License. Read Full License 


\section{Abstract}

Background: Intraspinal upper cervical C1-C2 tumors pose a challenge in resection. Internal fixation has routinely been used to prevent the occurrence of instability after atlantoaxial laminectomy for resection of C1-C2 intraspinal tumors, that sacrifices the motion of upper cervical spine. We therefore present this report to evaluate the efficacy and safety of one-stage posterior resection of these tumors through the atlantoaxial lamina space without internal fixation.

Methods: Ten suitably selected patient with C1-C2 intraspinal tumors were included in this study (period January 2016 to January 2018). All the patients underwent one-stage posterior resection through atlantoaxial lamina space without internal fixation. The efficacy of the procedure was documented by comparing postoperative and preoperative outcome scores [The visual analogue scores (VAS), Japanese Orthopedic Association scores (JOA), neck disability index (NDI)], cervical physiological curvature and range of flexion-extension. Safety was assessed by documenting the complications associated with surgery and subsequent sequale.

Results: 6 Male and 4 female patients with mean age 36 years (range 17 years to 50 years) underwent total tumor resection through posterior only approach using the atlantoaxial lamina space for the following tumors: 4 neurofibromas, 3 schwannomas and 3 meningiomas. The mean follow-up was 31.2 months (range 24-36 months). These patients' pathological types included. Postoperative VAS and NDI were lower than those of pre-operation with statistical significance $(p<0.05)$ while postoperative JOA was higher than that of pre-operation $(p<0.05)$. The physiological curvature and activity of cervical spine were maintained at latest follow up. Three patients suffered cerebrospinal fluid leakages that was managed consertaviley with no added intervention required. No patient had local recurrence at latest follow-up.

Conclusion: One-stage posterior resection through atlantoaxial lamina space without fixation is an effective and safe treatment for the upper cervical intraspinal tumor. In our experience this technique can remove tumor completely and does not cause instability to C1-C2 joint.

\section{Background}

Intraspinal cervical cord tumors are challenging [1], considering the complex anatomy and poor outcomes resulting from cervical cord compression. In addition to surgical decompression of the spinal cord, it is essential to completely resect the tumors and this remains the mainstay of treatment for tumors in this location [2, 3]. The preferred approach for tumors located posteriorly in the C1-C2 cervical spine involves posterior atlantoaxial laminectomy which provdes better surgical exposure and tumor access, but is associated with a risk of cervical instability and deformity $[4,5]$. Spinal internal fixation has routinely been used to prevent the occurrence of spinal instability, however it increases surgical time, instrumentation related injuries and limitation of function of upper cervical spine. 
As the distance between the inferior edge of the $\mathrm{C} 1$ posterior arch and the upper edge of the C2 lamina is relatively large and with our novel approach we aimed to exploit this anatomical window to gain access for surgical resection of posterior C1-C2 intraspinal tumors without destabilizing the C1-C2 complex and thus elimination the need for fixation. We report the safety and efficacy of this surgical approach with our experience of 10 patients.

\section{Methods}

\section{Patients}

Patients with intraspinal tumors of the upper cervical spine were screened at presentation for eligibility and willingness to undergo tumor surgery via the new surgical approach under study. All patients underwent a clinical examination and local imaging for tumor resection planning. MRI confirming extramedullary intraspinal tumors posteriorly located at C1-2 level and CT angiography (CTA) confirming the tumor was not adhesive to the vertebral artery were further analyzed for supero-inferior extent and tumors not exceeding the upper edge of C1 and/or lower edge of C2 lamina were considered for resection using the atlantoaxial lamina space approach. The lateral limiting factor was the C1-C2 lateral joint margin. The tumor was preliminarily judged as benign by MRI and CT showed no bony destruction.

Between January 1, 2016, and January 1, 2018, 10 patients were included for the study to undergo one-stage posterior resection operation and were included in the final analysis. At intial clinical examination, the most common presentation was neck pain $(80 \%, 8 / 10)$, followed by numbness $(40 \%$, 8/10), limb weakness $(20 \%$, $2 / 10)$, hypoesthesial $(10 \%, 1 / 10)$, and dizziness $(10 \%, 1 / 10)$. The study was approved by the ethics committee of General Hospital of Southern Theatre Command of PLA and this study was conducted in conformity with human principles of research..

\section{Preoperative Evaluation}

Local imaging included radiographs of cervical spine (standard and dynamic views), CT and contrast enhanced MRI. The stereoscopic images were depicted by three dimensional CT reconstructions. Vertebral artery angiography was used to assess the proximity of the tumor to the vertebral artery. The diameter of the tumor measured by MRI ranged from 1.8 to $3.2 \mathrm{~cm}$ in these ten patients (Table 1 ).

Table 1.Clinical Characteristics of the 10 patients with C1-2 intraspinal tumors 


\begin{tabular}{lllllll}
\hline Case & Gender & Age & MRI tumor diameter $(\mathrm{cm})$ & Tumor type & Symptoms & Follow-up \\
\multicolumn{1}{c}{ (years) } & & & & \\
(months) \\
\hline 1 & Male & 17 & 3.2 & Neurofibroma & Neck pain; numbness & 24 \\
2 & Female & 29 & 2.8 & Neurofibroma & Neck pain & 36 \\
3 & Female & 32 & 2.4 & Meningioma & Neck pain; hypoesthesia & 30 \\
4 & Male & 46 & 2.0 & Schwannoma & Upper limb weakness & 24 \\
5 & Female & 50 & 2.6 & Neurofibroma & Neck pain; numbness & 36 \\
6 & Male & 42 & 3.0 & Schwannoma & Neck pain; dizziness & 36 \\
7 & Male & 34 & 2.1 & Meningioma & Neck pain; numbness & 30 \\
8 & Female & 36 & 2.2 & Meningioma & Lower limb weakness & 24 \\
9 & Male & 48 & 1.8 & Neurofibroma & Neck pain & 36 \\
10 & Male & 26 & 2.8 & Schwannoma & Neck pain; numbness & 36 \\
\hline
\end{tabular}

\section{Surgical Procedures}

General anesthesia was performed with tracheal intubation. After the patient was positioned prone, the skull traction weighing 3-4 kg was applied to maintain neck in moderate flexion position to enlarge the lamina space of C1-C2. Then, the skin was longitudinally incised along the posterior midline of the neck from the occipital to the C2 spinous process. Subperiosteal dissection in both directions was performed to expose the occipital bone, the posterior arch of $\mathrm{C} 1$, the lower edge of $\mathrm{C} 2$ lamina and the lateral mass joint of $\mathrm{C} 1-\mathrm{C} 2$ without damaging the joint capsule to fully expose the lamina space of C1-C2. The posterior cervical muscular attachment points were not damaged, and extensor muscle insertion on the C2 spinous process and the C1 arch was preserved. If the tumor was intradural, the dura mater should be cut open and pulled to two sides using surgical suture. After the tumor was sutured and suspended gradually, the tumor capsule was carefully separated followed by removal of the tumor (Fig. 1). The spinal cord and nerve root were protected carefully. Then, the dura mater was sutured tightly and covered with repair material to prevent cerebrospinal fluid leakage. If the tumor was extradural, it was sutured and suspended gradually to separate tumor capsule microscopically followed by removal of the tumor. If the tumor was closely adhered to the nerve root, the proximal nerve root which was connected to the tumor was cut before complete removal of the tumor. After a drainage tube was placed, the incision was sutured 
in layers. The tumor was sent to pathological examination. All patients' pathological types were all verified by pathological examination after operation.

\section{Postoperative management and follow-up}

The vital signs were closely monitored after operation. Prophylactic use of antibiotics, appropriate use of glucocorticoids and neurotrophic drugs were administered as necessary. Postoperative scores were analysed at 1month,1year and 2years following surgery. The neural function was evaluated by JOA [6] score (17 points method) and neck disability index (NDI) [8]. The level of neck pain was evaluated by VAS [7] score. The cervical X-ray was reexamined to measure the cervical curvature and activity after operation. MRI was also retested to understand the tumor removal. The neck bracket was used for 4 weeks. Post operative rehabilitation protocol included at postoperative 1 month and followed by full range of activity at 24 months. The follow-up was in the form of outpatient reexamination at 1 month, 1 year and 2 years after operation included clinical examination and local imaging.

\section{Statistical Analysis}

Data was entered into a spreadsheet and SPSS Version 22.0 (IBM, Chicago IL, USA) statistical software package was used for statistical analysis. The Kolmogorov-Smirnov test $(P>0.10)$ was used for testing normality distribution of quantitative variables. The mean and standard deviation (SD) was used for expressing normally distributed data. The repeated measure ANOVA was used investigate changes in mean VAS score, JOA score and NDI, as well as physiological curvature and activity of cervical spine at different following up time. The post HOC statistical analysis was used for multiple comparisons. A two tailed $\mathrm{P}$ value $<0.05$ was considered statistically significant.

\section{Results}

Six male and 4 female patients with mean age 36 years (range 17 years to 50 years) underwent total tumor resection through posterior only approach using the atlantoaxial lamina space for the following tumors: 4 neurofibromas, 3 schwannomas and 3 meningiomas. Minimum follow up was 24 months before considering analysis of results. Mean follow up was 31.2 months (range 24 to 36 months). The 
surgical time ranged from 110 to $220 \mathrm{~min}$, with an average duration of $161.00 \pm 34.78 \mathrm{~min}$. Total surgical blood loss ranged from 100 to $800 \mathrm{ml}$, with an average of $410.00 \pm 202.48 \mathrm{ml}$. All the surgeries were carried out under the monitoring of somatosensory evoked potential and no significant decrease of evoked potential was found during the operation. Extensive hemorrhage or vertebral artery injury was not observed in our study group. Postoperative MRI confirmed complete tumor removal as achieved in all patients and 2 patients required transection of the ipsilatera $\mathrm{C} 2$ root to allow separation of the tumor. Immediate postoperative improvement in clinical parameters: neck pain 8 patients, Numbness 4 patients and return of power 2 patient was observed. Fig. 2 showed significant improvement in VAS, NDI and JOA scores at latest follow up when compared to the preoperative scoring $(p<0.05)$. There was no significant difference in physiological curvature of the cervical spine or the range of flexion-extension at latest follow up when compares to the preoperative measurements ( $p>0.05 ;$ Fig. 3 ). There were 3 patients with cerebrospinal fluid leakage postoperatively that were managed by conservative approach. No other complications were observed intraoperatively or in the postoperative period. No local recurrence has been observed at last follow up (Fig. 4).

\section{Discussion}

C1-C2 intraspinal tumors accounted for $5 \%-12 \%$ of all spinal nerve root tumors and $18 \%-30 \%$ of all cervical nerve root tumors [9-11]. Resection operation is often required to remove the tumors. In order to remove the tumors completely to a large extent at a low risk, more C1-C2 structures are damaged in posterior resection, which has a risk of C1-C2 instability.

Surgical stabilization is commonly performed after the removal of tumors with the purpose of preventing the occurrence of $\mathrm{C} 1-\mathrm{C} 2$ instability. Posterior $\mathrm{C} 1-\mathrm{C} 2$ and $\mathrm{C0}-\mathrm{C} 2$ fixation and fusion techniques are widely used. But, these managements sacrifice the normal motion of the C1-C2 joints, and the incidence of degeneration of the subaxial cervical spine may be increased.

Due to the relatively large distance from the $\mathrm{C} 1$ posterior arch to the upper edge of the $\mathrm{C} 2$ lamina, in this study, we tried to remove C1-C2 intraspinal tumor through this space without destroying the C1-C2 stable structures, and fixation was also unused. The clinical efficacy and safety of this technique were evaluated. To our knowledge, this is the first time to report a one-stage posterior resection through atlantoaxial lamina space without internal fixation for C1-C2 intraspinal tumor.

Our results demonstrate one-stage posterior resection through atlantoaxial lamina space is effective in the treatment of $\mathrm{C} 1-\mathrm{C} 2$ intraspinal tumor. We found that the VAS score was lower than that before surgery. This proved that the patients got good comfort because of this new operation, which achieved the same treatment effect as the traditional operation [12-13]. Meanwhile, the increased scores of JOA indicates that this new operation can remove the tumor completely and reduce the compression of the spinal cord or nerve root, relieving the numbness symptoms of the patients, improving the muscle strength. The most vital facet was that we kept the cervical physiological curve and range of flexionextension to the greatest extent. According to the Asazuma et al. [14] study, if the operation segment 
involved the $\mathrm{C} 2$, the cervical physiological curve and range of flexion-extension would decrease after four year's follow-up. In the last follow-up of this study, the cervical X-ray showed no increase in the angle and curvature of the cervical spine, which was related to the fact that we did not damage the C1-C2 stable structures during the operation, and was beneficial to the recovery of the patients.

The efficacy of this new technique can be also seen from the low incidence of tumor recurrence. After 2 year's follow-up, no local recurrence occurred in all patients in our study. We consider that this may be related to the complete resection of the tumor under the open surgery. Compared with the intraspinal tumor resection under the microscope, the latter is more minimally invasive, but the possibility of tumor recurrence is relatively high, which is due to incomplete tumor resection under microscope [15] .

Our results illustrate this new technique is also safe in the treatment of C1-C2 intraspinal tumor. Before the operation, we carried out CT and MRI of the upper cervical spine to verify the relationship between the tumor and the surrounding soft tissue, and CTA was obtained to identify whether there was adhesion between the tumor and the vertebral artery or not so that we tried our best to avoid the risk of massive hemorrhage and acute cerebral infarction caused by the injury of the vertebral artery. Finally, there was no injury to spinal cord or blood vessels during operation. Although 3 patients suffered cerebrospinal fluid leakage after operation, they were cured 2 weeks after operation with active handling. During our followup, no signs of C1-C2 instability and other complications associated with surgery was found. Beside, strict admission criteria and the consent of patients and their families are also essential facets to ensure the safety of the operation.

\section{Limitation}

First, due to the strict inclusion criteria and exclusion criteria, as well as whether the patients agreed to accept this new operation or not, we finally just included 10 patients. In view of the safety and effectiveness of the operation, we are now trying our best to accumulate the numbers of cases. Secondly, MRI could be reexamined in order to better reassess the change of the patients' upper cervical spine. Thirdly, a much longer follow-up might be needed to verify the efficacy and safety of this new technique.

\section{Conclusion}

One-stage posterior resection through atlantoaxial lamina space without fixation is a effective and safe treatment of the upper cervical intraspinal tumor, which can provide complete removal of tumor, and preserves stability of C1-C2 joint.

\section{Abbreviations}

CT: Computed tomography; CTA: Computed tomography angiography; MRI: Magnetic resonance imaging; VAS: Visual analog scale; JOA: Japanese Orthopedic Association scores; NDI: Neck disability index 


\section{Declarations}

Ethics approval and consent to participate

This study was approved by the ethics committee of General Hospital of Southern Theatre Command of PLA and the written informed consent for participation in the study was obtained.

\section{Availability of data and materials}

The data used and analyzed during the current study are available in anonymized form from the corresponding author on reasonable request.

\section{Competing interests}

The authors declare that they have no competing interests.

\section{Funding}

This work was supported by the Guangzhou Municipal Science and Technology Project (No. 201803010046); the National Natural Science Foundation of China (No. 81672232); and the Science and Technology Planning Project of Guangdong Province (No. 2015B020233013).

\section{Authors' contributions}

MX designed the study and critically revised the manuscript. OB, ZX, NL carried out the statistical analyses, and drafted the manuscript. GS, CY, ZS were responsible for the data collection and measurement of radiographic data. XH checked the manuscript. All authors read and approved the final manuscript.

\section{Acknowledgements}

We would like to thank Jianhua Wang, Kai Zhang for guidance of measurement of radiographic data. 
${ }^{1}$ The First School of Clinical Medicine, Southern Medical University, No.1838 North of Guangzhou Road, Guangzhou, 510515, People's Republic of China. ${ }^{2}$ Guangzhou University of Traditional Chinese Medicine, No.12 of Airport Road, Guangzhou, 510006, People's Republic of China. ${ }^{3}$ Department of Orthopedics, General Hospital of Southern Theatre Command of PLA, 111 Liuhua Road, Guangzhou, 510010, People's Republic of China.

\section{References}

1. Laminoplasti K. Laminoplasty using the Posterior Midline Approach in the Treatment of C1-2 Spinal Tumors. Turk Neurosurg. 2015; 25: 595-600.

2. Nakamura M, Iwanami A, Tsuji O. Long-term surgical outcomes of cervical dumbbell neurinomas. Journal of Orthopaedic Science. 2013; 18: 8-13.

3. Matsumoto Y, Harimaya K, Kawaguchi K. Dumbbell scoring system: a new method for the differential diagnosis of malignant and benign spinal dumbbell tumors. Spine, 2016; 41: E1230-E1236.

4. Wang X, Gao J, Wang T. The Long-term Outcome After Resection of Upper Cervical Spinal Cord Tumors: Report of 51 Consecutive Cases. Scientific reports. 2018; 8: 1.

5. Wang Y, Lou P, Li M. Clinical features and surgical procedures of intradural-epidural and epidural cervical dumbbell tumors. Chinese medical journal. 2013; 126:2797- 2798.

6. Yonenobu K, Abumi K, Nagata K, Taketomi E, Ueyama K. Interobserver and intraobserver reliability of the Japanese Orthopaedic Association scoring system for evaluation of cervical compression myelopathy. Spine. 2001;26:1890-1895.

7. Zanoli G, Strömqvist B, Jönsson B. Visual analog scales for interpretation of back and leg pain intensity in patients operated for degenerative lumbar spine disorders. Spine. 2001;26:2375-2380.

8. Swanenburg J, Humphreys K, Langenfeld A, Brunner F, Wirth B. Validity and reliability of a German version of the Neck Disability Index (NDI-G). Man Ther. 2014;19:52-58.

9. Maurya P, Singh K, Sharma V. C1 and C2 nerve sheath tumors: Analysis of 32 cases. Neurol India. 2009;57: 31-3.

10. George B, Lot G. Neurinomas of the first two cervical nerve roots: A series of 42 cases. J Neurosurg. 1995;82: 917-923.

11. Krishnan P, Behari S, Banerji D, Mehrotra N, Chhabra DK, Jain VK. Surgical approach to C1-C2 nerve sheath tumors. Neurol India. 2004;52: 319-324.

12. Zong S, Zeng G, Xiong C. Treatment results in the differential surgery of intradural extramedullary schwannoma of 110 cases. PloS one. 2013; 8: e63867.

13. Ahn DK, Park HS, Choi DJ. The surgical treatment for spinal intradural extramedullary tumors. Clinics in orthopedic surgery. 2009; 1: 165-172.

14. Asazuma T, Toyama Y, Maruiwa H. Surgical strategy for cervical dumbbell tumors based on a threedimensional classification. Spine. 2004; 29: E10-E14. 
15. Yu Y, Zhang X, Hu F. Minimally invasive microsurgical treatment of cervical intraspinal extramedullary tumors. Journal of Clinical Neuroscience. 2011; 18: 1168-1173.

\section{Figures}

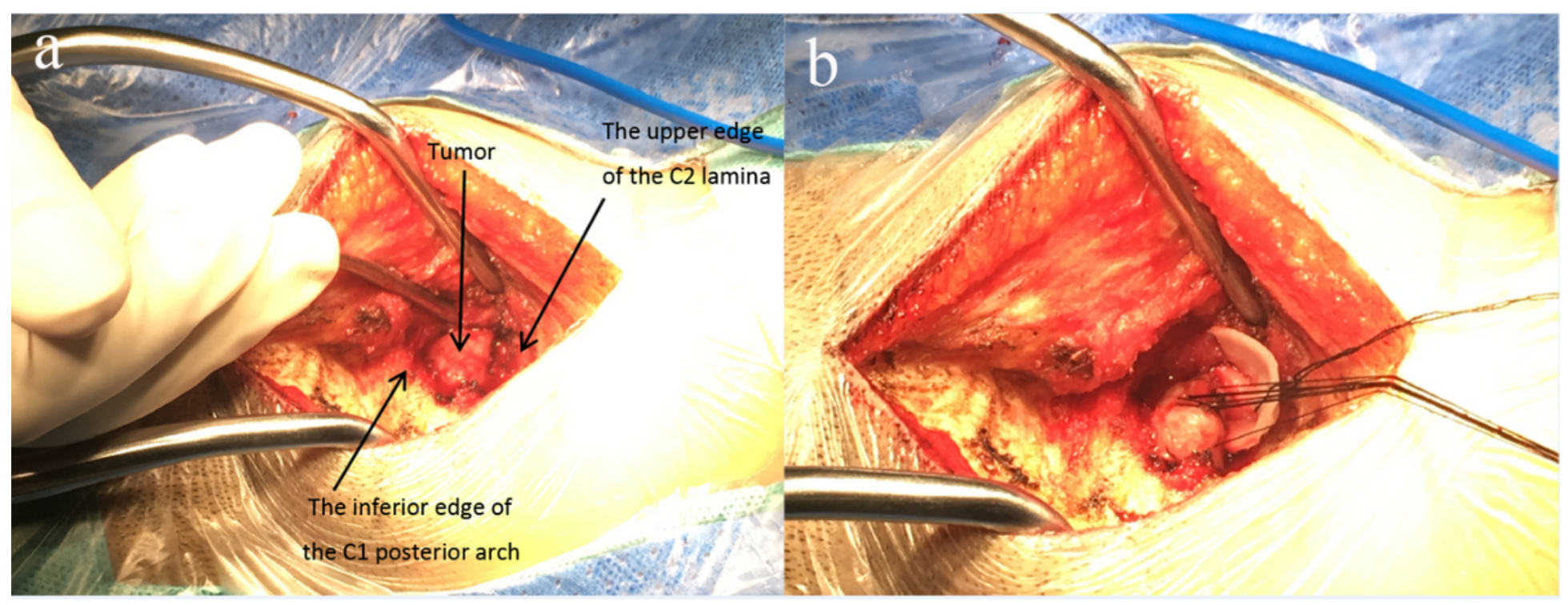

\section{Figure 1}

During the operation, the tumors in atlantoaxial lamina space were exposed (a), suspended by sutures (b), and excised after careful stripping

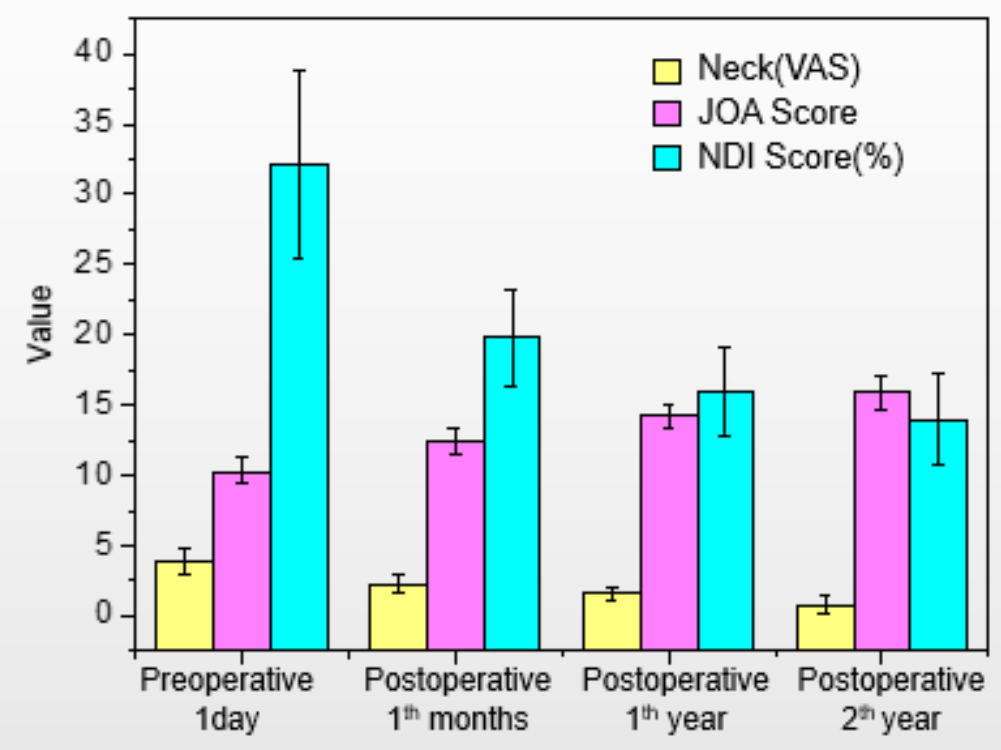

\section{Figure 2}

Comparison of VAS, JOA score, NDI score at different time 


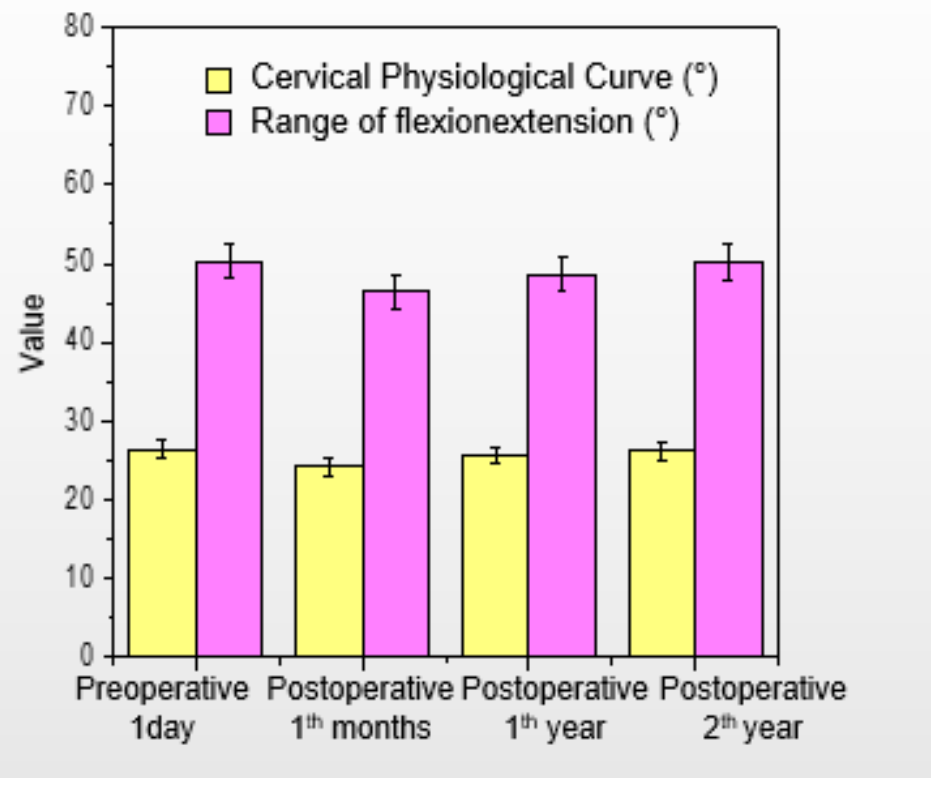

\section{Figure 3}

Comparison of physiological curvature and range of flexion-extension of cervical spine at different time
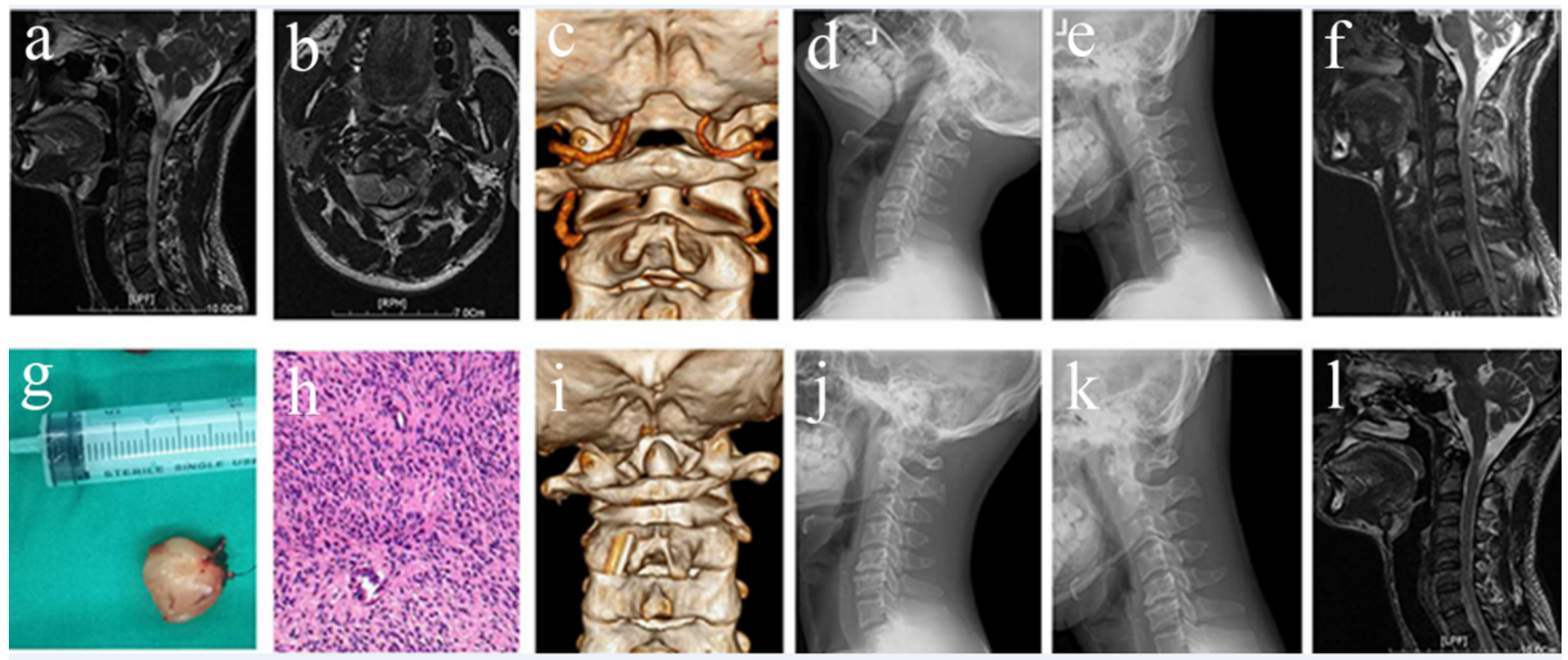

\section{Figure 4}

A 48-year-old male patient was diagnosed with C1-C2 intraspinal tumor. a and b. Preoperative T2weighted MR images showed C1-C2 intraspinal tumor. c. Preoperative 3D CT scan with CTA showed no adhesion between vertebral artery and tumor. $d$ and e. Preoperative cervical dynamic X-rays showed no C1-C2 instability. f. Postoperative 1th months sagittal T2-weighted MRI image showed that the tumor was removed completely. g. Excised tumor. h. Pathological examination showed the tumor was neurofibroma i. Postoperative 1th months 3D CT scan showed the integrity of atlantoaxial structures. j and k. Postoperative 2th years cervical dynamic X-ray showed no C1-C2 instability and normal cervical 
physiological curve. I. Postoperative 2th years sagittal T2-weighted MRI image showed no recurrence of the tumor 